\title{
Using BIM model for Fire Emergency Evacuation Plan
}

\author{
Adam Stančík ${ }^{1, *}$, Roman Macháček ${ }^{2}$, and Jiř́ Horák ${ }^{1}$ \\ ${ }^{1}$ Brno University of Technology, Institute of Computer Aided Engineering and Computer Science, 602 \\ 00 Brno, Veveri 331/95, Czech Republic \\ ${ }^{2}$ Czech Technical University in Prague, Department of Steel and Timber Structures, 16636 Prague 6, \\ Czech Republic
}

\begin{abstract}
Is it possible to use building information modelling (BIM) to create a fire emergency evacuation plan in the national condition of the Czech Republic? This article focuses on the advantages and possibilities of using BIM for fire protection.
\end{abstract}

\section{Introduction}

When there is a fire, every second counts. Fire and smoke spread very fast. To avoid unnecessary loss of human life, it is important to have a well-thought out fire evacuation plan. But that is not the only requirement. For better orientation within a burning building, a floor plan with marked escape routes that everyone is familiar with is created. This plan is the graphic part of a fire evacuation plan. For a fire evacuation plan to be effective, it must be kept up to date throughout the service life of the building. This is what building information modelling (BIM) can do for fire emergency evacuation plans.

\section{Preparation for BIM model}

Currently there are no specifications for BIM in the Czech Republic. This has resulted in there being a big difference in the quality of various models. These models are frequently created for investors for future use, for example for facility management; however the investors often do not know themselves what to expect and see nothing more in the BI model than a 3D view of the final state of the object. Those with more experience know that they should focus more on the information aspect of the model. The basic elements (in Autodesk Revit(C BIM software these elements are called 'families') of these models can sometimes be found in several world languages, from common languages such as English, German and French to Chinese, which is harder to translate. Unfortunately, this linguistic diversity appears not only in non-reported parameters, but also in reported parameters. The model designers themselves are usually familiar with the model, however there are situations where

\footnotetext{
*Corresponding author: stancik.a@,fce.vutbr.cz
} 
the creators revisit the model after a period of time and are unsure what specific items were intended for. Linguistic consistency should, therefore, be one of the basic features of a model. The language for the whole project should be clearly defined at the beginning of a project. In the case of BIM, this should at least be the case for the reported parameters.

\section{Cooperation}

Many professions - and people who are forced to communicate with each other - enter the lifecycle of a building. To facilitate communication, BIM sharing information using a single building information model, as shown in Figure 1 on the left. In the Figure 1 on the right, one person has been added. This person is often not spoken about within the BI model, but this person is an inherent part of every building, whether it is a family home or the tallest building in the world. That person is the designer of the building's fire safety solution - the fire engineer. It is the fire engineer who ensures the safety of the people using the proposed building. The designer of a building's fire safety solution is involved in several stages of the building's lifecycle:

1. Planning - the creation of a fire safety solution (PBR), including the design of escape routes and the calculation of evacuation time;

2. Implementation - the creation of necessary reports and the location of fire safety equipment (PBZ);

3. Building operation (control) - PBZ revision and control, and possible future use of data for rescue units in case of a fire in the building.
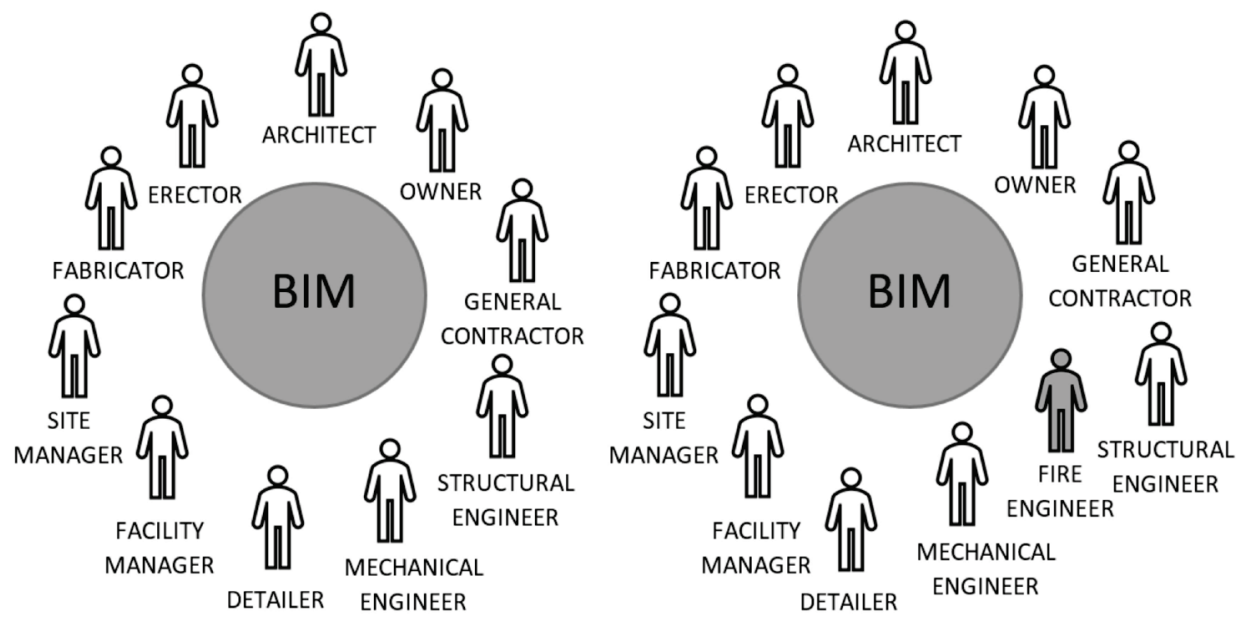

Fig. 1. The professions involved in the lifecycle of a building.

\section{Parameterization}

The information BIM provides is much more valuable than the actual 3D interpretation of objects in the model. The 3D view can enable us to know that there is an extinguisher in the building, however this 3D view can also be enhanced by the addition of an information folder that provides the localization, exact type, manufacturer, price, fire class, type of dispenser, temperature range, fire capabilities and amount of extinguishing media, as well as a picture 
of the device and, last but not least, all the information that is generally stated on the label of a fire extinguisher, such as the year of production, and the inspection and revision dates. Each bit of information is understood as a parameter for the element or 'family'. The number of element parameters is unlimited. All information is reportable and can be used not only in project documentation or construction, but also in the management of the building. How detailed the information is depends on the requirements of the investor and the authors of the model. Parameters can be further sorted into groups for better clarity. Parameters can be numeric, integral or of the YES/NO type, among other options. Most of the parameters we create and use are text-based. Other than using them for reporting, it is also possible to use these parameters to create a graphic display of a fire plan.

\section{Families}

As mentioned earlier, the model consists of basic elements called 'families'. The model created in the BIM Revit $\subseteq$ tool normally consists of all three family types: system-based, model-based and embedded. Creating families is one of the advanced controls of the abovementioned software. In the beginning, the goal was to process the signs used to create the fire evacuation plans of a building and then use them to create these plans. Example of some of these signs are shown in Figure 2. The following basic rules are used in the formation of families:

- It is a combination of 2D symbol and $3 \mathrm{D}$ object.

- The insertion point is located at the one edge of the symbol.

- The family is 'cleaned up' and all unnecessary items, such as unused materials, lines and hatches, are removed as they affect the resulting file size.

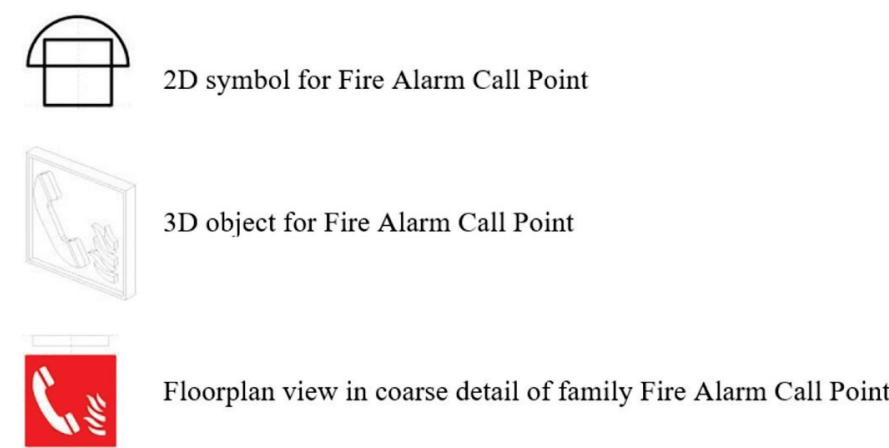

Fig. 2. Example of one of signs used for evacuation fire plans.

\section{Evacuation fire plans}

Article 33 of Decree No. 246/2001 Coll. on fire prevention states what must be included in a given fire evacuation plan. A fire evacuation plan consists of both text and graphics, to which we are obligated by the following point of the above-mentioned decree: " $f$ ) graphical representation of the direction of escape routes on individual floors" [1]. The graphical portion of an evacuation plan is prepared in the form of a floor plan on which the individual floors of the building are plotted out, as well as escape routes and directions, fire extinguishers, fire hydrants, fire alarms, the fire assembly point and the safety instructions in 
case of a fire or accident. The graphical map of the fire evacuation plan must also include the position in which the observer is currently located. When structural changes are made to a building, fire evacuation plans can become outdated, and this can have fatal consequences. This problem could be solved by having a maintained BI model as the basis for processing the graphical part of a building's fire evacuation plan itself. On the graphical portion of a fire evacuation plan, apart from the floor plan, which can be updated, there are parts of the plan that do not need to be changed and are, thus, valid for all future plans. These still-valid elements include the instructions in the event of a fire, the instructions in the event of an accident and the explanation of what the signs in the fire evacuation plan mean. This is done by means of legends, which are copied on to the drawings of the evacuation plans for each individual floor.

\section{Creating the graphical portion of the fire evacuation plan}

The actual creation of the graphical portion of the fire evacuation plan is partly dependent on the data used by the architect or civil engineer. With a few adjustments, we can accomplish an extremely clear layout of an evacuation plan in a short space of time, while maintaining the maximum information necessary for the safe evacuation of building occupants. These adjustments include: setting a single solid hatch line for walls, leaving only the numbers and names of rooms, turning off the detailed display of the fillings of openings such as windows and doors, and leaving only empty openings and a number of other modifications. To complete the graphical portion of the fire evacuation plan, it is necessary to fill in the ground plan with the above-mentioned legend. Additionally, a sign with the exact location of the fire evacuation plan must be added to the plan.

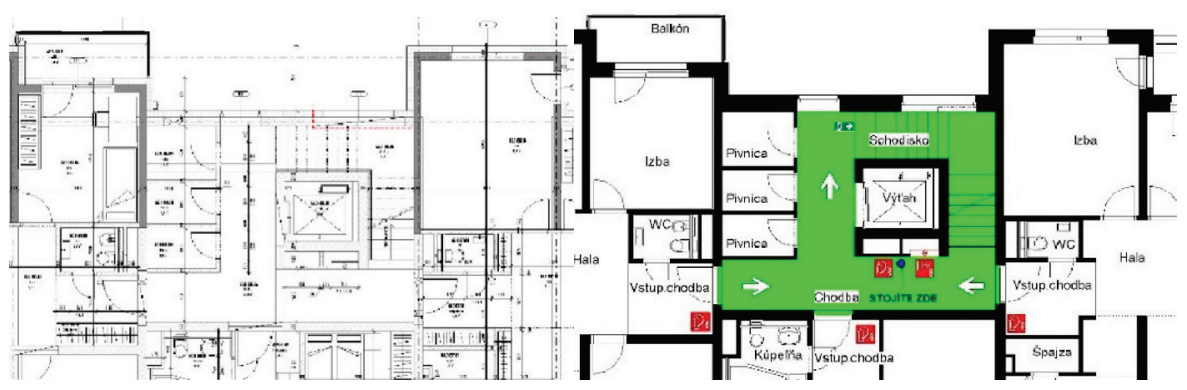

Fig. 3. Part of the evacuation plan before (left side) and after (right side) editing the ground plan of the building.

\section{Add-on application and plug-in for BIM software}

Almost all tasks designers of fire safety solutions undertake when designing project documentation can be programmed in order to reduce the time the work takes, especially when it comes to repeat processes. In this case, there are a few simple commands that, in their entirety, make up a complex code. BIM software Revit $\subset$ provides a rich and powerful .NET API that can be used to automate repetitive tasks, and extend the core functionality of Revit in simulation, conceptual design, construction and building management, and much more. It is possible to program Revit's .NET API with any .NET compliant language, including VB.NET, C\# and C++/CLI. The Software Development Toolkit (SDK) provides 
extensive .NET code samples and documentation to assist the beginning stages of development with Revit's API [2]. In addition, an application that would save fire safety solution designers a lot of work, and in the best cases managed to generate 'drawings' for the fire evacuation plan by itself, is in the development stages.

\section{Information model and visual programming}

For those who are interested in creating their own tools, but are limited by the programming language, the standard BIM parameters of the software can be extended by an add-on for visual programming that uses an algorithmic graphics editor environment. The add-on has been developed as an open source project, allowing developers and users to actively participate in its development and continual improvement. The main difference between code writing and visual programming is that visual programming uses the associated nodes to perform the given operation.

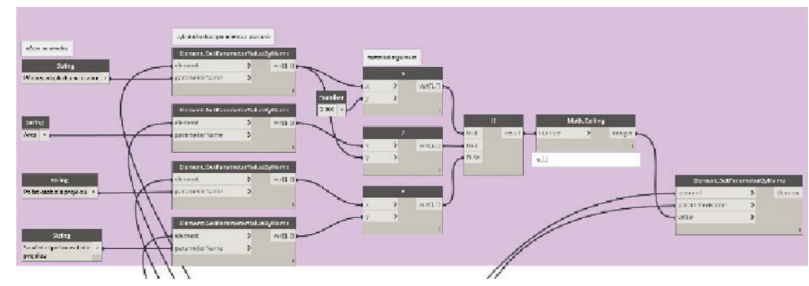

Fig. 4. A script assigning the correct values using the logic function.

There is an unlimited number of possible uses for visual programming. Designers of fire safety solutions can be assisted with seemingly easy steps, as well as complicated ones, such as calculating the length of an escape route or the number of evacuated people.

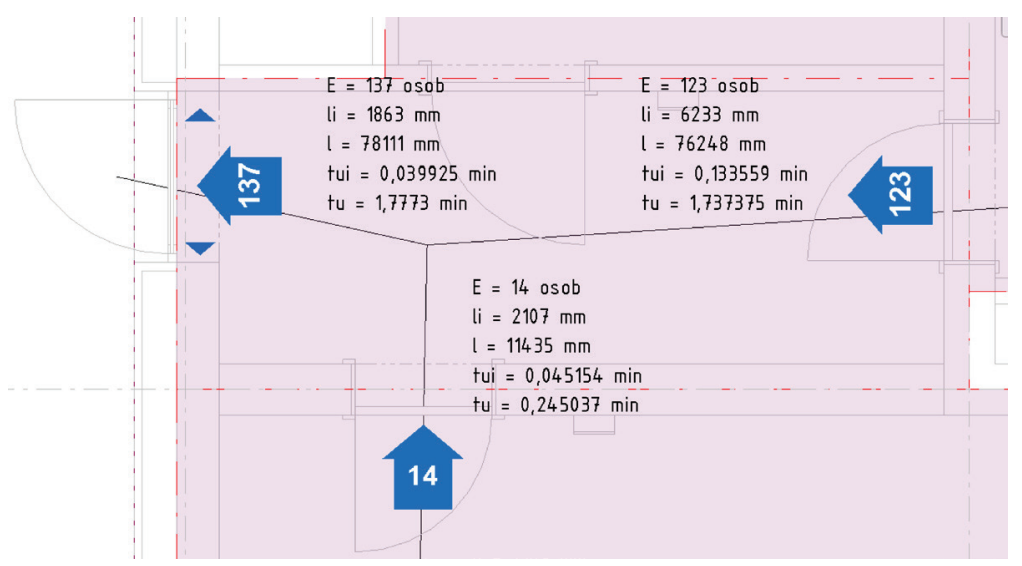

Fig. 5. Counting values from multiple consequential segments.

In this case, the designer would follow several steps. The first step for a designer using the tool is to create a new project that is joined to the building model that is linked to the original file. This ensures that the changes made in the original file will take effect in the new project as well. The next step is to select the function of individual rooms in the building. This automatically assigns values in order to help calculate the number of building occupants. Subsequently, each room is assigned to a fire compartment, where other values can be added 
for calculation and the persons in the individual rooms belonging to it can be added up. Finally, a network of escape routes, which represents the streams of exiting people towards the open area, is formed in the model. After defining the escape routes, the final step is to run a script created using the visual programming plug-in. This script will run a number of algorithms that primarily assign the number of people from the fire sectors, following which the number of evacuated persons is assigned to the first segment of the escape route. In case of the collision of multiple segments in one place, the script will decide on a greater value of evacuated persons. The algorithm is repeated several times before adding up the people in all of the segments. The script proceeds analogically when calculating the length of the escape route and the evacuation time. Calculated values; such as the length of the escape route, the number of evacuees and the evacuation time; can then be reported in a simple way and provide a comprehensive overview in order to assess the requirements. Using this data, if the report is unsuitable; for example, an escape door is too narrow, or an escape route is too long; the object can easily be edited and the calculation recommenced.

\section{Conclusion}

When there is a fire, every second counts. It is vital that fire evacuation plans are kept up to date. Thanks to BIM models and their subsequent use, we are able to pass on information quickly and with minimal loss. There is a higher requirement for fire safety solution designers to also have knowledge of information technology, but those who are fluent in programming languages or are able to use visual programming will save a lot of time on tasks that take up tens of minutes of an ordinary designer's time. In cases with a well working code, the error rate that could arise from the need to repeat the process several times during the creation of the plan can also be minimized.

Acknowledgement: This paper has been worked out under the project No. FAST-J-17-4590 and under the project No. FAST-S-17-4275

\section{References}

1. Act No. 246/2001 Coll., On determination of conditions of fire safety and execution of state fire surveillance (Fire Prevention Regulation) (Collection of Laws, Prague, 2001). Available on https://www.zakonyprolidi.cz/cs/2001-246

2. Autodesk Developer Network. Available on http://usa.autodesk.com/adsk/servlet/index?siteID $=123112 \& \mathrm{id}=2484975$ 